\title{
Association study of ACE and eNOS single nucleotide polymorphisms with Henoch-Schönlein purpura nephritis
}

\author{
BO DI ${ }^{1 *}$, XINYUAN LI ${ }^{2 *}$, LIQUN SONG $^{3}$, QIANGHU WANG ${ }^{4}$ and SUYAN LIU ${ }^{2}$ \\ ${ }^{1}$ The First Affiliated Hospital; ${ }^{2}$ The Second Affiliated Hospital, Harbin Medical University; \\ ${ }^{3}$ Heilongjiang University of Chinese Medicine; ${ }^{4}$ Department of Bioinformatics Science and Technology, \\ Harbin Medical University, Harbin, Heilongjiang 150080, P.R. China
}

Received April 5, 2012; Accepted August 2, 2012

DOI: $10.3892 / \mathrm{mmr} .2012 .1032$

\begin{abstract}
Previous studies have shown that insertion/deletion polymorphisms in the angiotensin-converting enzyme (ACE) gene and the endothelial nitric oxide synthase (eNOS) gene are associated with Henoch-Schönlein purpura nephritis (HSPN). However, further studies are required to prove the relationship between HSPN and ACE and eNOS single nucleotide polymorphisms (SNPs). We studied six ACE SNPs and two eNOS SNPs by genotyping HSPN patients. Statistical analyses indicate that four ACE SNPs and two eNOS SNPs are associated with HSPN susceptibility. A cumulative effect analysis suggested that an increased number of unfavourable genotypes may lead to an increased risk of HSPN. By comparing alleles, genotypes and haplotypes that are associated with lupus nephritis (LN) and HSPN, we found genetic heterogeneity between HSPN and LN.
\end{abstract}

\section{Introduction}

Henoch-Schönlein purpura (HSP) is an autoimmune disorder that is associated with environmental and genetic factors, such as infection and genetic polymorphisms in the associated genes (1-3). HSP may cause multisystem clinical symptoms including arthritis, skin rashes and abdominal pain (4). Renal injury is a serious complication that occurs in $33 \%$ of children and $63 \%$ of adults with HSP (5).

Previous studies have suggested that the angiotensin-converting enzyme (ACE) gene is associated with

Correspondence to: Professor Suyan Liu, The Second Affiliated Hospital, Harbin Medical University, No. 157 Baojian Road, Harbin, Heilongjiang 150080, P.R. China

E-mail: liusuyan_med@163.com

*Contributed equally

Key words: Henoch-Schönlein purpura nephritis, angiotensinconverting enzyme gene, endothelial nitric oxide synthase gene, single nucleotide polymorphisms, association
Henoch-Schönlein purpura nephritis (HSPN). Ozkaya et al and Zhou et al previously reported that an insertion/deletion polymorphism in the ACE gene is associated with $\operatorname{HSPN}(6,7)$. The ACE gene, which encodes for the enzyme that converts angiotensin I to angiotensin II, is expressed in numerous tissues and is important in regulating renal cell growth (8). HSP is a form of small vessel vasculitis that is characterised by mediation of the IgA immune complex $(9,10)$. Deposits of the $\operatorname{IgA}$ immune complex in the capillary walls may cause vasculitis, and if these deposits precipitate in the kidney, they may cause HSPN. ACE polymorphisms may affect angiotensin II regulation, which may lead to renal injury. Furthermore, ACE polymorphisms are associated with the risk of many other autoimmune diseases, such as $\operatorname{Ig} \mathrm{A}$ nephropathy and lupus nephropathy $(\mathrm{LN})(11,14)$. Therefore, SNPs in the ACE gene may be a genetic component of HSPN. We also chose to study the association between endothelial nitric oxide synthase (eNOS) and HSPN. eNOS plays a role in nitric oxide (NO) synthesis (15), which is associated with autoimmune-mediated tissue destruction and autoimmune diseases, such as systemic lupus erythematosus (SLE) and experimental autoimmune encephalomyelitis $(16,17)$. Moreover, eNOS also plays an important role in vascular disease pathogenesis.

Although several studies have focused on the association between the previously described ACE gene insertion/deletion polymorphism and HSPN occurrence and severity, as well as the association between NO and the occurrence of SLE, few studies have analysed the association between ACE and eNOS polymorphisms with HSPN. However, previous studies have analysed ACE and eNOS gene polymorphisms in association with LN by studying six ACE SNPs (A-5466C, T-3892C, A-240T, C1237T, G2215A and A2350G) and two eNOS SNPs (T-786C and G894T). As SLE and HSP are autoimmune diseases that commonly result in renal injury, we analysed these eight SNPs to study the relationship between ACE and eNOS gene polymorphisms and HSPN $(10,18)$. In addition to studying the relationship between ACE and eNOS polymorphisms and HSPN, we also sought to explore the genetic heterogeneity of LN and HSPN. This study will provide information about the molecular mechanisms of HSPN disease pathogenesis and may provide information that will aid in HSPN clinical diagnosis and treatment. 


\section{Materials and methods}

Subjects. The control samples were obtained from healthy consenting blood donors from the northern Chinese population, and the HSPN samples were obtained from hospitalised renopathy patients. All the patients were selected according to the following criteria: i) a history of skin purpura and kidney damage, with or without symptoms involving the gastrointestinal tract and joints; ii) a renal biopsy pathology corresponding to HSPN; iii) the exclusion of systemic diseases, such as thrombocytopenic purpura, SLE, systemic vasculitis and rheumatoid arthritis; and iv) no history of treatment with large doses of hormones or immunosuppressive agents, such as cyclophosphamide. Samples where the specific locus of interest could not be genotyped were excluded from the analysis. Therefore, our final data set consisted of 160 case samples (out of 229 patients) and 137 control samples (out of 218 controls). The case and control samples were age- and gender-matched. All research adhered to the legal requirements of the study country. The study was approved by the ethics committee of Harbin Medical University. The investigators educated potential subjects to ensure that they could reach an informed decision about whether or not to participate in the research. Their informed consent was given freely, without coercion, and based on a clear understanding of what participation involves.

SNP genotyping. A description of the eight SNPs (six ACE gene and two eNOS gene polymorphisms) used in our analysis is shown in Table I. After the DNA was extracted from the blood samples, the DNA quality was analysed by electrophoresis. PCR amplification was performed for each locus, and the detailed information for the PCR reaction and cycling conditions for each locus is shown in Table II.

Statistical analysis. After we genotyped the HSPN and control subjects, the distribution of the genotypes was directly computed. The Hardy-Weinberg equilibrium test was performed for all eight SNPs in the control group. The association analysis of the genotypes and HSPN was performed by logistic regression, and the risk alleles were determined by a Chi-squared test. In addition, the association between the G894T genotype and HSPN was analysed with a Chi-squared test. The odds ratio (OR) and the associated $95 \%$ confidence interval (CI) were computed for each genotype.

Haplotype analysis of the six ACE SNPs and the two eNOS SNPs was performed with HaploView. We obtained the haplotypes and the estimated frequency of each haplotype for the case and control groups with HaploView. We then performed a Chi-squared test to analyse the association between each haplotype and HSPN. If the number of haplotypes was fewer than 5, we then used Fisher's exact test to calculate the p-value and assess significance.

We also analysed the cumulative effect of the unfavourable genotypes. An unfavourable genotype was defined as a genotype that was significantly associated $(\mathrm{p}<0.05)$ with HSPN and had a corresponding OR greater than 1 . We divided the disease and control samples into three groups according to the number of unfavourable genotypes in an individual. The groups are: 2 or fewer unfavourable genotypes; 3-4 unfavourable genotypes; and 5 or more unfavourable genotypes. We defined the 2 or fewer unfavourable genotypes group as the reference and compared this group to the other two groups with a Chi-squared test.

\section{Results}

Genotype distribution of ACE and eNOS and the association between these polymorphisms and HSPN. The genotype frequencies of each ACE SNP (A2350G, A-5466C, C1237T, A-240T, A2215G and T-3892C) are shown in Table III. The frequencies in the case group were 23.13 (AA), 70.00 (AG) and $6.87 \%(\mathrm{GG})$ for A2350G; 9.38 (AA), $32.50(\mathrm{AC})$ and $58.12 \%$ (CC) for A-5466C; 11.25 (CC), 55.63 (CT) and 33.13\% (TT) for C1237T; 10.63 (AA), 81.88 (AT) and 7.50\% (TT) for A-240T; 32.50 (AA), 23.75 (AG) and 43.75\% (GG) for A2215G; and 11.88 (TT), 43.75 (TC) and $44.37 \%$ (CC) for T-3892C. In the control group, the values were 18.25 (AA), 53.28 (AG) and $28.47 \%$ (GG) for A2350G; 3.65 (AA), 40.15 (AC) and 56.20\% (CC) for A-5466C; 10.95 (CC), 49.64 (CT) and 39.42\% (TT) for C1237T; 7.30 (AA), 61.31 (AT) and 31.39\% (TT) for A-240T; 35.04 (AA), 13.14 (AG) and 51.82\% (GG) for A2215G and 18.25 (TT), 27.74 (TC) and 54.01\% (CC) for T-3892C. Three loci (A-240T, A2215G and T-3892C) that were not in Hardy-Weinberg equilibrium in the control subjects were also included in our analysis.

As is shown in Table III, the A2350G AG ( $p=0.003$; $\mathrm{OR}=2.046 ; 95 \% \mathrm{CI}=1.270-3.294)$ and $\mathrm{GG}(\mathrm{p}=0.000 ; \mathrm{OR}=0.186$; 95\% CI=0.091-0.380) genotypes, A-240T AT $(\mathrm{p}=0.000$; $\mathrm{OR}=2.850 ; 95 \% \mathrm{CI}=1.679-4.838)$ and $\mathrm{TT}(\mathrm{p}=0.000 ; \mathrm{OR}=0.177$; 95\% CI=0.089-0.353) genotypes, A2215G AG genotype $(\mathrm{p}=0.021 ; \mathrm{OR}=2.059 ; 95 \% \mathrm{CI}=1.113-3.809)$ and $\mathrm{T}-3892 \mathrm{C} \mathrm{TC}$ genotype $(p=0.005 ; O R=2.026 ; 95 \% C I=1.245-3.299)$ were distributed significantly differently in the case and control subjects. By contrast, there was no difference in the distribution of the other ACE genotypes between HSPN patients and the control subjects ( $p>0.05$, Table I). Additionally, the A240 allele $(\mathrm{p}=0.001 ; \mathrm{OR}=1.739 ; 95 \% \mathrm{CI}=1.253-2.415)$ and the A2350 allele $(\mathrm{p}=0.001$; $\mathrm{OR}=1.704 ; 95 \% \mathrm{CI}=1.230-2.358)$ were significantly associated with HSPN.

The two eNOS SNPs (G894T and T-786C) were in Hardy-Weinberg equilibrium in the control group. The G894T GT $(\mathrm{p}=0.000 ; \mathrm{OR}=0.339 ; 95 \% \mathrm{CI}=0.284-0.404)$ and TT genotypes $(\mathrm{p}=0.000 ; \mathrm{OR}=3.025 ; 95 \% \mathrm{CI}=2.526-3.623)$, as well as the T-786C TT $(\mathrm{p}=0.001 ; \mathrm{OR}=2.779 ; 95 \% \mathrm{CI}=1.484-5.207)$ and TC genotypes $(\mathrm{p}=0.034 ; \mathrm{OR}=0.600 ; 95 \% \mathrm{CI}=0.374-0.962)$ were distributed significantly differently between the HSPN and control subjects. In addition, the G894T and T-786C alleles were significantly associated with $\operatorname{HSPN}(\mathrm{p}=0.000$ and $\mathrm{p}=0.022$, respectively).

ACE and eNOS haplotype distribution and the association between these variations and HSPN. We manually obtained 33 ACE gene haplotypes with HaploView and analysed the haplotype distribution of the six SNPs in the case and control subjects. As is shown in Table IV, the CCTTGG $(\mathrm{p}=0.012 ; \mathrm{OR}=0.517$; 95\% CI $=0.307-0.873)$, CCTTAG $(\mathrm{p}=0.023$; OR=0.536; $95 \%$ $\mathrm{CI}=0.311-0.923), \mathrm{CCACAA}(\mathrm{p}=0.013 ; \mathrm{OR}=2.604 ; 95 \% \mathrm{CI}=1.198$ 5.658), CTATAA ( $\mathrm{p}=0.024 ; \mathrm{OR}=0.319 ; 95 \% \mathrm{CI}=0.112-0.906)$ and ACTCGA haplotypes ( $\mathrm{p}=0.040 ; \mathrm{OR}=0.347 ; 95 \% \mathrm{CI}=0.121-$ $0.996)$ were significantly different $(p<0.05)$ in the case and control groups. Moreover, only the CCACAA haplotype $(\mathrm{OR}>1)$ is more common in the HSPN group than in the control group. 
Table I. Description of the six ACE gene and two eNOS gene SNPs.

\begin{tabular}{|c|c|c|c|c|}
\hline SNP & Primer sequence & Enzyme & $\begin{array}{l}\text { Amplification } \\
\text { length (bp) }\end{array}$ & $\begin{array}{l}\text { Length of digested } \\
\text { fragments (bp) }\end{array}$ \\
\hline \multirow[t]{2}{*}{ ACE (A-5466C) } & F-gccatgtcacatgtattatagga & & & \\
\hline & R-cgtctttggaaacttgtctgc & EcoRV & 133 & $24+109$ \\
\hline \multirow[t]{2}{*}{ ACE (T-3892C) } & F-atagtgtatatagggcttggtac & & & \\
\hline & R-agaagatatttgcaaagtatgtactg & Pst $\mathrm{I}$ & 114 & $90+24$ \\
\hline \multirow[t]{2}{*}{$\mathrm{ACE}(\mathrm{A}-240 \mathrm{~T})$} & F-tgtcactccggaggcgggaggct & & & \\
\hline & R-gagaaagggectcctctctct & $X b a \mathrm{I}$ & 168 & $145+23$ \\
\hline \multirow[t]{2}{*}{$\operatorname{ACE}(\mathrm{C} 1237 \mathrm{~T})$} & F-agtgcacacgggtcacgatg & & & \\
\hline & R-caccaagtagccaaagggcag & BsmBI & 287 & $95+192$ \\
\hline \multirow[t]{2}{*}{ ACE (G2215A) } & F-cacaccctgaagtacggcac & & & \\
\hline & R-tcctccagctcctgggcag & HaeII & 131 & $109+22$ \\
\hline \multirow[t]{2}{*}{ ACE (A2350G) } & F-ctgacgaatgtgatggccgc & & & \\
\hline & R-ttgatgagttccacgtatttcg & $B s t \mathrm{UI}$ & 122 & $103+19$ \\
\hline \multirow[t]{2}{*}{ eNOS (T-786C) } & F-tggagatgatggtgtacccca & & & \\
\hline & R-gectccacceccaccetgtc & MspI & 180 & $92+46+42$ \\
\hline \multirow[t]{2}{*}{ eNOS (G894T) } & F-aaggcaggagacagtggatgga & & & \\
\hline & R- cccagtcaatccetttggtgetca & BanII & 248 & $163+85$ \\
\hline
\end{tabular}

ACE, angiotensin-converting enzyme; eNOS, endothelial nitric oxide synthase; SNP, single nucleotide polymorphism; F, forward; R, reverse.

Table II. PCR reaction and cycling conditions for each locus.

\begin{tabular}{|c|c|c|}
\hline SNP & PCR reaction components & PCR cycling conditions \\
\hline ACE (A-240T) & $\begin{array}{l}2 \mathrm{X} \text { buffer } 10 \mu \mathrm{l} \text {; Taq-E } 0.5 \mu \mathrm{l} ; 10 \mathrm{~m} \text { dNTPs } 0.5 \mu \mathrm{l} \text {; } \\
10 \mathrm{M} \text { each primer } 1 \mu \mathrm{l} \text {; DNA } 4 \mu \mathrm{l} \text {; } \mathrm{H}_{2} \mathrm{O} 3 \mu \mathrm{l} \text {; Total } 19 \mu \mathrm{l}\end{array}$ & $\begin{array}{l}94^{\circ} \mathrm{C} 5 \mathrm{~min} ; 94^{\circ} \mathrm{C} 30 \mathrm{sec} ; 60^{\circ} \mathrm{C} 30 \mathrm{sec} ; 35 \text { cycles; } \\
72^{\circ} \mathrm{C} 30 \mathrm{sec} ; 72^{\circ} \mathrm{C} 5 \mathrm{~min}\end{array}$ \\
\hline ACE (A2350G) & $\begin{array}{l}2 \mathrm{X} \text { buffer } 10 \mu \mathrm{l} \text {; Taq-E } 0.5 \mu \mathrm{l} ; 10 \mathrm{M} \text { dNTPs } 0.5 \mu \mathrm{l} \text {; } \\
10 \mathrm{M} \text { each primer } 1 \mu \mathrm{l} \text {; DNA } 2 \mu \mathrm{l} \text {; } \mathrm{H}_{2} \mathrm{O} 5 \mu \mathrm{l} \text {; Total } 19 \mu \mathrm{l}\end{array}$ & $\begin{array}{l}94^{\circ} \mathrm{C} 5 \mathrm{~min} ; 94^{\circ} \mathrm{C} 30 \mathrm{sec} ; 60^{\circ} \mathrm{C} 30 \mathrm{sec} ; 10 \text { cycles; } \\
72^{\circ} \mathrm{C} 30 \mathrm{sec} ; 94^{\circ} \mathrm{C} 30 \mathrm{sec} ; 50^{\circ} \mathrm{C} 30 \mathrm{sec} ; 30 \text { cycles; } \\
72^{\circ} \mathrm{C} 30 \mathrm{sec} ; 72^{\circ} \mathrm{C} 5 \mathrm{~min}\end{array}$ \\
\hline ACE (A-5466C) & $\begin{array}{l}2 \mathrm{X} \text { buffer } 10 \mu \mathrm{l} \text {; Taq-E } 0.5 \mu \mathrm{l} ; 10 \mathrm{M} \text { dNTPs } 0.5 \mu \mathrm{l} \text {; } \\
10 \mathrm{M} \text { each primer } 1 \mu \mathrm{l} \text {; DNA } 2 \mu \mathrm{l} \text {; } \mathrm{H}_{2} \mathrm{O} 5 \mu \mathrm{l} \text {; Total } 19 \mu \mathrm{l}\end{array}$ & $\begin{array}{l}94^{\circ} \mathrm{C} 5 \mathrm{~min} ; 94^{\circ} \mathrm{C} 30 \mathrm{sec} ; 50^{\circ} \mathrm{C} 30 \mathrm{sec} ; 35 \text { cycles; } \\
72^{\circ} \mathrm{C} 30 \mathrm{sec} ; 72^{\circ} \mathrm{C} 5 \mathrm{~min}\end{array}$ \\
\hline ACE (A2215G) & $\begin{array}{l}2 \mathrm{X} \text { buffer } 10 \mu \mathrm{l} \text {; Taq-E } 0.5 \mu \mathrm{l} ; 10 \mathrm{M} \text { dNTPs } 0.5 \mu \mathrm{l} \text {; } \\
10 \mathrm{M} \text { each primer } 1 \mu \mathrm{l} \text {; DNA } 2 \mu \mathrm{l} \text {; } \mathrm{H}_{2} \mathrm{O} 5 \mu \mathrm{l} \text {; Total } 19 \mu \mathrm{l}\end{array}$ & $\begin{array}{l}94^{\circ} \mathrm{C} 5 \mathrm{~min} ; 94^{\circ} \mathrm{C} 30 \mathrm{sec} ; 60^{\circ} \mathrm{C} 30 \mathrm{sec} ; 35 \text { cycles; } \\
72^{\circ} \mathrm{C} 30 \mathrm{sec} ; 72^{\circ} \mathrm{C} 5 \mathrm{~min}\end{array}$ \\
\hline ACE (T-3892C) & $\begin{array}{l}2 \mathrm{X} \text { buffer } 10 \mu \mathrm{l} \text {; Taq-E } 0.5 \mu \mathrm{l} ; 10 \mathrm{M} \text { dNTPs } 0.5 \mu \mathrm{l} \text {; } \\
10 \mathrm{M} \text { each primer } 1 \mu \mathrm{l} \text {; DNA } 2 \mu \mathrm{l} \text {; } \mathrm{H}_{2} \mathrm{O} 5 \mu \mathrm{l} \text {; Total } 19 \mu \mathrm{l}\end{array}$ & $\begin{array}{l}94^{\circ} \mathrm{C} 5 \mathrm{~min} ; 94^{\circ} \mathrm{C} 30 \mathrm{sec} ; 50^{\circ} \mathrm{C} 30 \mathrm{sec} ; 35 \text { cycles; } \\
72^{\circ} \mathrm{C} 30 \mathrm{sec} ; 72^{\circ} \mathrm{C} 5 \mathrm{~min}\end{array}$ \\
\hline $\mathrm{ACE}(\mathrm{C} 1237 \mathrm{~T})$ & $\begin{array}{l}2 \mathrm{X} \text { buffer } 10 \mu \mathrm{l} \text {; Taq-E } 0.5 \mu \mathrm{l} ; 10 \mathrm{M} \text { dNTPs } 0.5 \mu \mathrm{l} \text {; } \\
10 \mathrm{M} \text { each primer } 1 \mu \mathrm{l} \text {; DNA } 2 \mu \mathrm{l} \text {; } \mathrm{H}_{2} \mathrm{O} 5 \mu \mathrm{l} \text {; Total } 19 \mu \mathrm{l}\end{array}$ & $\begin{array}{l}94^{\circ} \mathrm{C} 5 \mathrm{~min} ; 94^{\circ} \mathrm{C} 30 \mathrm{sec} ; 60^{\circ} \mathrm{C} 30 \mathrm{sec} ; 10 \text { cycles; } \\
72^{\circ} \mathrm{C} 30 \mathrm{sec} ; 94^{\circ} \mathrm{C} 30 \mathrm{sec} ; 50^{\circ} \mathrm{C} 30 \mathrm{sec} ; 30 \text { cycles; } \\
72^{\circ} \mathrm{C} 30 \mathrm{sec} ; 72^{\circ} \mathrm{C} 5 \mathrm{~min}\end{array}$ \\
\hline eNOS (G894T) & $\begin{array}{l}2 \mathrm{X} \text { buffer } 10 \mu \mathrm{l} \text {; Taq-E } 0.5 \mu \mathrm{l} ; 10 \mathrm{M} \text { dNTPs } 0.5 \mu \mathrm{l} \text {; } \\
10 \mathrm{M} \text { each primer } 1 \mu \mathrm{l} \text {; DNA } 2 \mu \mathrm{l} \text {; } \mathrm{H}_{2} \mathrm{O} 5 \mu \mathrm{l} \text {; Total } 19 \mu \mathrm{l}\end{array}$ & $\begin{array}{l}94^{\circ} \mathrm{C} 5 \mathrm{~min} ; 94^{\circ} \mathrm{C} 30 \mathrm{sec} ; 60^{\circ} \mathrm{C} 30 \mathrm{sec} ; 10 \text { cycles; } \\
72^{\circ} \mathrm{C} 30 \mathrm{sec} ; 94^{\circ} \mathrm{C} 30 \mathrm{sec} ; 50^{\circ} \mathrm{C} 30 \mathrm{sec} ; 30 \text { cycles; } \\
72^{\circ} \mathrm{C} 30 \mathrm{sec} ; 72^{\circ} \mathrm{C} 5 \mathrm{~min}\end{array}$ \\
\hline eNOS (T-786C) & $\begin{array}{l}2 \mathrm{X} \text { buffer } 10 \mu \mathrm{l} \text {; Taq-E } 0.5 \mu \mathrm{l} \text {; } 10 \mathrm{M} \text { dNTPs } 0.5 \mu \mathrm{l} \text {; } \\
10 \mathrm{M} \text { each primer } 1 \mu \mathrm{l} \text {; DNA } 2 \mu \mathrm{l} \text {; } \mathrm{H}_{2} \mathrm{O} 5 \mu \mathrm{l} \text {; Total } 19 \mu \mathrm{l}\end{array}$ & $\begin{array}{l}94^{\circ} \mathrm{C} 5 \mathrm{~min} ; 94^{\circ} \mathrm{C} 30 \mathrm{sec} ; 60^{\circ} \mathrm{C} 30 \mathrm{sec} ; 10 \text { cycles; } \\
72^{\circ} \mathrm{C} 30 \mathrm{sec} ; 94^{\circ} \mathrm{C} 30 \mathrm{sec} ; 50^{\circ} \mathrm{C} 30 \mathrm{sec} ; 30 \mathrm{cycles} ; \\
72^{\circ} \mathrm{C} 30 \mathrm{sec} ; 72^{\circ} \mathrm{C} 5 \mathrm{~min}\end{array}$ \\
\hline
\end{tabular}

ACE, angiotensin-converting enzyme; eNOS, endothelial nitric oxide synthase; SNP, single nucleotide polymorphism.

Haplotype analysis of the eNOS gene indicated that the two eNOS polymorphism loci are closely linked. Three haplotypes were identified with HaploView. As shown in Table V, the frequency of these three haplotypes was significantly different 
Table III. The SNP distribution of the ACE and eNOS genotypes and alleles.

\begin{tabular}{|c|c|c|c|c|c|}
\hline SNP & Genotype & Control $n=137(\%)$ & Case $n=160(\%)$ & P-value & OR $(95 \% \mathrm{CI})$ \\
\hline \multirow[t]{5}{*}{$\mathrm{ACE}(\mathrm{A}-240 \mathrm{~T})$} & 1(AA) & $10(7.30)$ & $17(10.63)$ & 0.323 & $1.510(0.667-3.417)$ \\
\hline & $2(\mathrm{AT})$ & $84(61.31)$ & $131(81.88)$ & $0.000^{\mathrm{a}}$ & $2.850(1.679-4.838)$ \\
\hline & $3(\mathrm{TT})$ & $43(31.39)$ & $12(7.50)$ & $0.000^{\mathrm{a}}$ & $0.177(0.089-0.353)$ \\
\hline & A allele & $104(37.96)$ & $165(51.56)$ & $0.001^{\mathrm{a}}$ & $1.739(1.253-2.415)$ \\
\hline & $\mathrm{T}$ allele & $170(62.04)$ & $155(48.44)$ & $0.001^{\mathrm{a}}$ & $0.575(0.414-0.798)$ \\
\hline \multirow[t]{5}{*}{ ACE (A2350G) } & $1(\mathrm{AA})$ & $25(18.25)$ & $37(23.13)$ & 0.304 & $1.348(0.763-2.379)$ \\
\hline & $2(\mathrm{AG})$ & $73(53.28)$ & $112(70.00)$ & $0.003^{\mathrm{a}}$ & $2.046(1.270-3.294)$ \\
\hline & $3(\mathrm{GG})$ & $39(28.47)$ & $11(6.87)$ & $0.000^{\mathrm{a}}$ & $0.186(0.091-0.380)$ \\
\hline & A allele & $123(44.89)$ & $186(58.13)$ & $0.001^{\mathrm{a}}$ & $1.704(1.230-2.358)$ \\
\hline & G allele & $151(55.11)$ & $134(41.87)$ & $0.001^{\mathrm{a}}$ & $0.587(0.424-0.813)$ \\
\hline \multirow[t]{5}{*}{ ACE (A-5466C) } & $1(\mathrm{AA})$ & $5(3.65)$ & $15(9.38)$ & 0.058 & $2.731(0.966-7.721)$ \\
\hline & $2(\mathrm{AC})$ & $55(40.15)$ & $52(32.50)$ & 0.172 & $0.718(0.446-1.155)$ \\
\hline & $3(\mathrm{CC})$ & $77(56.20)$ & $93(58.12)$ & 0.739 & $1.082(0.682-1.715)$ \\
\hline & A allele & $65(23.72)$ & $82(25.63)$ & 0.592 & $1.107(0.762-1.610)$ \\
\hline & C allele & $209(76.28)$ & $238(74.38)$ & 0.592 & $0.903(0.621-1.313)$ \\
\hline \multirow[t]{5}{*}{ ACE (A2215G) } & $1(\mathrm{AA})$ & $48(35.04)$ & $52(32.50)$ & 0.645 & $0.893(0.551-1.446)$ \\
\hline & $2(\mathrm{AG})$ & $18(13.14)$ & $38(23.75)$ & $0.021^{\mathrm{a}}$ & $2.059(1.113-3.809)$ \\
\hline & $3(\mathrm{GG})$ & $71(51.82)$ & $70(43.75)$ & 0.165 & $0.723(0.457-1.143)$ \\
\hline & A allele & $114(41.61)$ & $142(44.38)$ & 0.497 & $1.120(0.808-1.550)$ \\
\hline & G allele & $160(58.39)$ & $178(55.62)$ & 0.497 & $0.893(0.645-1.237)$ \\
\hline \multirow[t]{5}{*}{ ACE (T-3892C) } & 1(TT) & $25(18.25)$ & $19(11.88)$ & 0.126 & $0.604(0.316-1.152)$ \\
\hline & 2(TC) & $38(27.74)$ & $70(43.75)$ & $0.005^{\mathrm{a}}$ & $2.026(1.245-3.299)$ \\
\hline & $3(\mathrm{CC})$ & $74(54.01)$ & $71(44.37)$ & 0.098 & $0.679(0.429-1.074)$ \\
\hline & $\mathrm{T}$ allele & 88 (32.12) & $108(33.75)$ & 0.673 & $1.076(0.764-1.517)$ \\
\hline & C allele & $186(67.88)$ & $212(66.25)$ & 0.673 & $0.929(0.659-1.309)$ \\
\hline \multirow[t]{5}{*}{$\mathrm{ACE}(\mathrm{C} 1237 \mathrm{~T})$} & $1(\mathrm{CC})$ & $15(10.95)$ & $18(11.25)$ & 0.934 & $1.031(0.498-2.132)$ \\
\hline & $2(\mathrm{CT})$ & $68(49.64)$ & $89(55.63)$ & 0.303 & $1.272(0.805-2.010)$ \\
\hline & 3(TT) & $54(39.42)$ & $53(33.13)$ & 0.261 & $0.761(0.473-1.224)$ \\
\hline & $\mathrm{C}$ allele & 98 (35.77) & $125(39.06)$ & 0.408 & $1.151(0.824-1.608)$ \\
\hline & $\mathrm{T}$ allele & $176(64.23)$ & $195(60.94)$ & 0.408 & $0.869(0.622-1.213)$ \\
\hline \multirow[t]{5}{*}{ eNOS (G894T) } & $1(\mathrm{GG})$ & $3(2.19)$ & $0(0)$ & 0.097 & - \\
\hline & $2(\mathrm{GT})$ & $55(40.15)$ & $0(0)$ & $0.000^{\mathrm{a}}$ & - \\
\hline & $3(\mathrm{TT})$ & $79(57.66)$ & $160(100)$ & $0.000^{\mathrm{a}}$ & - \\
\hline & G allele & $61(22.26)$ & $0(0)$ & $0.000^{\mathrm{a}}$ & - \\
\hline & T allele & $213(77.74)$ & $320(100)$ & $0.000^{\mathrm{a}}$ & - \\
\hline \multirow[t]{5}{*}{ eNOS (T-786C) } & $1(\mathrm{TT})$ & $16(11.68)$ & $43(26.88)$ & $0.001^{\mathrm{a}}$ & $2.779(1.484-5.207)$ \\
\hline & 2(TC) & $61(44.53)$ & $52(32.50)$ & $0.034^{\mathrm{a}}$ & $0.600(0.374-0.962)$ \\
\hline & $3(\mathrm{CC})$ & $60(43.80)$ & $65(40.63)$ & 0.581 & $0.878(0.553-1.394)$ \\
\hline & $\mathrm{T}$ allele & $93(33.94)$ & $138(43.13)$ & $0.022^{\mathrm{a}}$ & $1.475(1.057-2.062)$ \\
\hline & $\mathrm{C}$ allele & $181(66.06)$ & $182(56.88)$ & $0.022^{\mathrm{a}}$ & $0.678(0.485-0.946)$ \\
\hline
\end{tabular}

aindicates alleles or genotypes with a p-value $<0.05$. ACE, angiotensin-converting enzyme; eNOS, endothelial nitric oxide synthase.

$(\mathrm{p}<0.05)$ in the HSPN and control subjects. The association between the ACE and eNOS haplotypes and HSPN was analysed with a Chi-squared test. If the number of the haplotypes was fewer than 5, we then used Fisher's exact test value to calculate a p-value and analyse significance.

Cumulative effect of ACE and eNOS unfavourable genotypes on HSPN. The six SNPs [A-240T (AT), A2350G (AG), A2215G (AG), T-3892C (TC), G894T (TT) and T-786C
(TT)] were analysed with a cumulative effect analysis. The frequency of five ACE genotypes [A-240T (AT), A2350G (AG), A2215G (AG), T-3892C (TC) and T-786C (TT)] was significantly different $(\mathrm{p}<0.05)$ between the case and control groups, and the OR values were greater than 1. Additionally, the proportion of the G894T phenotype (TT) in the case group was significantly higher than the control group. Therefore, we defined G894T (TT) as an unfavourable genotype. The subjects were divided into groups based on their number of 
Table IV. Haplotype analysis of the ACE gene.

\begin{tabular}{|c|c|c|c|c|}
\hline Haplotype & Case $2 \mathrm{n}=320(\%)$ & Control $2 \mathrm{n}=274(\%)$ & P-value & OR (95\%CI) \\
\hline CCTTGG & $26(8.13)$ & $40(14.60)$ & $0.012^{\mathrm{a}}$ & $0.517(0.307-0.873)$ \\
\hline CCTTAG & $24(7.50)$ & $36(13.14)$ & $0.023^{\mathrm{a}}$ & $0.536(0.311-0.923)$ \\
\hline CCACAA & $26(8.13)$ & $9(3.28)$ & $0.013^{\mathrm{a}}$ & $2.604(1.198-5.658)$ \\
\hline CCACGA & $16(5.00)$ & $13(4.74)$ & 0.885 & $1.057(0.499-2.238)$ \\
\hline CCATAA & $18(5.63)$ & $8(2.92)$ & 0.108 & $1.982(0.848-4.632)$ \\
\hline CTACGA & $14(4.38)$ & $10(3.65)$ & 0.654 & $1.208(0.528-2.765)$ \\
\hline CTTTGA & $13(4.06)$ & $8(2.92)$ & 0.452 & $1.408(0.575-3.449)$ \\
\hline CTATGA & $12(3.75)$ & $6(2.19)$ & 0.269 & $1.740(0.644-4.700)$ \\
\hline CTATAA & $5(1.56)$ & $13(4.74)$ & $0.024^{\mathrm{a}}$ & $0.319(0.112-0.906)$ \\
\hline ACTCGA & $5(1.56)$ & $12(4.38)$ & $0.040^{\mathrm{a}}$ & $0.347(0.121-0.996)$ \\
\hline CCATGG & $10(3.13)$ & $6(2.19)$ & 0.483 & $1.441(0.517-4.017)$ \\
\hline ATATGG & $10(3.13)$ & $4(1.46)$ & 0.278 & $2.177(0.675-7.022)$ \\
\hline ССТТАA & $10(3.13)$ & $3(1.09)$ & 0.157 & $2.914(0.794-10.697)$ \\
\hline CCTCGG & $6(1.88)$ & $7(2.55)$ & 0.572 & $0.729(0.242-2.195)$ \\
\hline CTTCAA & $8(2.50)$ & $4(1.46)$ & 0.400 & $1.731(0.515-5.811)$ \\
\hline CCTTGA & $6(1.88)$ & $6(2.19)$ & 0.786 & $0.854(0.272-2.677)$ \\
\hline CCTCAG & $8(2.50)$ & $4(1.46)$ & 0.400 & $1.731(0.515-5.811)$ \\
\hline ATTCGG & $4(1.25)$ & $6(2.19)$ & 0.525 & $0.565(0.158-2.025)$ \\
\hline CCTCGA & $4(1.25)$ & $7(2.55)$ & 0.361 & $0.483(0.140-1.667)$ \\
\hline ACTTGG & $4(1.25)$ & $5(1.82)$ & 0.739 & $0.681(0.181-2.562)$ \\
\hline CCACGG & $5(1.56)$ & $4(1.46)$ & 1.000 & $1.071(0.285-4.030)$ \\
\hline CTTTGG & $6(1.88)$ & $3(1.09)$ & 0.516 & $1.726(0.428-6.968)$ \\
\hline CCACAG & $6(1.88)$ & $3(1.09)$ & 0.516 & $1.726(0.428-6.968)$ \\
\hline ATATGA & $6(1.88)$ & $2(0.73)$ & 0.298 & $2.599(0.520-12.982)$ \\
\hline ACACAA & $6(1.88)$ & $2(0.73)$ & 0.298 & $2.599(0.520-12.982)$ \\
\hline CTTTAG & $3(0.94)$ & $5(1.82)$ & 0.481 & $0.509(0.121-2.150)$ \\
\hline ACATGA & $7(2.19)$ & $1(0.36)$ & 0.075 & $6.105(0.746-49.936)$ \\
\hline CTTCAG & $4(1.25)$ & $3(1.09)$ & 1.000 & $1.143(0.254-5.154)$ \\
\hline ACTTAG & $3(0.94)$ & $4(1.46)$ & 0.709 & $0.639(0.142-2.879)$ \\
\hline ATTTGA & $4(1.25)$ & $4(1.46)$ & 1.000 & $0.854(0.212-3.449)$ \\
\hline CTATAG & $1(0.31)$ & $6(2.19)$ & 0.053 & $0.140(0.017-1.170)$ \\
\hline CCATGA & $5(1.56)$ & $2(0.73)$ & 0.460 & $2.159(0.415-11.216)$ \\
\hline ACACGA & $3(0.94)$ & $3(1.09)$ & 1.000 & $0.855(0.171-4.270)$ \\
\hline
\end{tabular}

${ }^{\mathrm{a}} \mathrm{P}<0.05$. ACE, angiotensin-converting enzyme.

unfavourable genotypes, and the group with 2 or fewer unfavourable genotypes was defined as the reference. Compared to the reference, the group with 3-4 unfavourable genotypes has a higher odds risk $(\mathrm{OR}=4.528)$, while the group with 5 or more unfavourable genotypes has an even higher OR value $(\mathrm{OR}=61.923)($ Table VI).

Heterogeneity analysis between $L N$ and HSPN. LN and purpura nephritis are immune complex-mediated diseases that often result in renal injury. Additionally, it has been reported that LN may co-occur with immune thrombocytopenic purpura (19). We compared the genetic polymorphisms associated with LN and HSPN to study the genetic heterogeneity between LN and HSPN. For this analysis, we utilised LN data from our previous study (20), in which we showed that two ACE alleles (A-5466 and A2350) and one eNOS allele (G894) were associated with lupus nephropathy susceptibility. In comparison, two ACE alleles (A-240 and A2350) and one eNOS allele (T-786) were associated with HSPN susceptibility (Table VII).

Analysis of the alleles associated with HSPN and LN indicates that G894, A2350 and A5466 are unfavourable alleles for LN, while T786, A2350 and A240 are unfavourable alleles for HSPN. Thus, these results indicate that A2350 is a risk allele for both HSPN and LN. The genotype analysis indicates that the A2350G AG (A2350G) genotype may be associated with HSPN susceptibility, although this genotype may also protect individuals from LN. Moreover, five haplotypes (CCTTGG, CCTTAG, CCACAA, CTATAA and ACTCGA) were distributed significantly differently in the case and 
Table V. Haplotype analysis of the eNOS gene.

\begin{tabular}{lcccc}
\hline Haplotype & Case $2 \mathrm{n}=320(\%)$ & Control 2n=274 $(\%)$ & P-value & OR (95\% CI) \\
\hline CT & $182(56.88)$ & $181(66.06)$ & $0.022^{\mathrm{a}}$ & $0.678(0.485-0.946)$ \\
TT & $138(43.12)$ & $32(11.68)$ & $0.000^{\mathrm{a}}$ & $5.734(3.730-8.815)$ \\
TG & $0(0)$ & $61(22.26)$ & $0.000^{\mathrm{a}}$ & - \\
\hline
\end{tabular}

${ }^{\mathrm{a}} \mathrm{P}<0.05$. eNOS, endothelial nitric oxide synthase.

Table VI. Cumulative effect of ACE and eNOS unfavourable genotypes on HSPN.

\begin{tabular}{lccccc}
\hline $\begin{array}{l}\text { Number of } \\
\text { unfavourable genotypes }\end{array}$ & Case (n) & Control (n) & OR & $95 \%$ CI & P-value \\
\hline 2 or fewer & 26 & 70 & 1 (reference) & & \\
$3-4$ & 111 & 66 & 4.528 & $2.629-7.798$ & $0.000^{\text {a }}$ \\
5 or more & 23 & 1 & 61.923 & $7.955-482.022$ & $0.000^{\mathrm{a}}$ \\
\hline
\end{tabular}

${ }^{a} \mathrm{P}<0.05$. ACE, angiotensin-converting enzyme; eNOS, endothelial nitric oxide synthase; HSPN, Henoch-Schönlein purpura nephritis.

Table VII. Genetic heterogeneity between LN and HSPN.

\begin{tabular}{|c|c|c|c|c|c|}
\hline \multicolumn{2}{|c|}{ Associated alleles } & \multicolumn{2}{|c|}{ Associated genotypes } & \multicolumn{2}{|c|}{ Associated haplotypes } \\
\hline $\mathrm{LN}$ & HSPN & $\mathrm{LN}$ & HSPN & $\mathrm{LN}$ & HSPN \\
\hline T894(+), G894(-) & T786(+), C786(-) & A2350G: AA(+), AG(-) & A2350G: AG(+), GG(-) & CTTCGA(-) & CCTTGG(-) \\
\hline A2350(+), G2350(-) & A2350(+), G2350(-) & G894T: GG(+), GT(-) & G894T: GT(?), TT(?) & ATTCGA(-) & $\operatorname{ACATGG}(+)$ \\
\hline \multirow[t]{4}{*}{ A5466(+), C5466(-) } & $\mathrm{T} 240(+), \mathrm{A} 240(-)$ & A-5466C: AA(+), CC(-) & A-240T: AT(+), TT(-) & CCTTAG(-) & ACTCGA(-) \\
\hline & & & A2215G: AG(+) & $\operatorname{ACTTAA}(+)$ & $\operatorname{ACACGG}(+)$ \\
\hline & & & T-3892C: TC(+) & CTATAA(-) & $\mathrm{CCACAA}(+)$ \\
\hline & & & T-786C: TT(+), TC(-) & & \\
\hline
\end{tabular}

(+) Indicates that the allele/genotype/haplotype is unfavourable $(\mathrm{p}<0.05$ and $\mathrm{OR}>1)$, and $(-)$ indicates that the allele/genotype/haplotype $(\mathrm{p}<0.05$ and $\mathrm{OR}<1)$ will protect an individual from the indicated disease. (?) Indicates that the favourability of the genotype is unknown. LN, lupus nephritis; HSPN, Henoch-Schönlein purpura nephritis.

control groups. Of these haplotypes, CCACAA may increase HSPN susceptibility, and the other four haplotypes may protect individuals from HSPN. Similarly, three haplotypes (ACTTAA, ACACGG and ACATGC) may increase the risk of $\mathrm{LN}$, and the other two haplotypes (ATTCGA and CTTCGA) may decrease the LN susceptibility. Although HSPN and LN both contribute to the onset of nephritis, they do not share any ACE gene haplotypes. All of the inconsistencies mentioned may be genetic factors that underlie the different clinical phenotypes of HSPN and LN.

\section{Discussion}

Approximately $40 \%$ of children with HSP may develop nephritis (10), and this proportion is higher in adults. Both HSP and SLE are vascular autoimmune diseases $(10,18,19)$ and SLE may occur in conjunction with other immune diseases, such as immune thrombocytopenic purpura (19). Many reports have suggested that ACE gene polymorphisms are associated with SLE and HSP $(6,8)$. The ACE enzyme converts angiotensin I into angiotensin II, which is associated with reno-vascular hypertension and renal disease (21). Therefore, the ACE gene may play a role HSPN. eNOS also plays an important role in the occurrence of vascular disease (22) and is associated with autoimmune disease (23).

Haplotype analysis indicates that five ACE gene haplotypes (CCTTGG, CCTTAG, CCACAA, CTATAA and ACTCGA) are significantly associated with HSPN. Of these five haplotypes, only CCACAA may increase the risk of HSPN. However, the six ACE SNPs were not closely linked in this analysis, and therefore this haplotype may require further validation. Three eNOS gene haplotypes (CT, TT and TG) were identified, and all three were associated with HSPN. eNOS gene haplotype analysis indicates that the two SNPs are closely linked. 
For the cumulative effect of unfavourable genotype analysis, we set the group with the least number of unfavourable genotypes (less than or equal to two unfavourable genotypes) as the reference. The group with a medium number of unfavourable genotypes (3-4 unfavourable genotypes) had a 4.528-fold increased risk of HSPN compared to the reference group, and the high-risk group (5 or more unfavourable genotypes) had a 61.923-fold increased risk.

A limitation of our study is that the samples we selected may contain some bias, which may explain why several of the ACE loci were not in Hardy-Weinberg equilibrium in the control subjects and why the six ACE SNPs were not closely linked. Furthermore, we did not consider genetic and environmental factors, which may impact on HSPN pathogenesis. However, by analysing the association between the ACE and eNOS polymorphisms and HSPN, we did identify SNPs and haplotypes that may be associated with HSPN. Additionally, our cumulative effect data indicate that high-risk unfavourable genotypes may increase the risk of HSPN. However, larger studies are required to further analyse the effect of these polymorphisms on HSPN.

In conclusion, our data suggest that four ACE gene SNPs (A-240T, A2350G, A2215G and T-3892C) and two eNOS gene SNPs (G894T and T-786C) are significantly associated with HSPN. Five haplotypes (CCTTGG, CCTTAG, CCACAA, CTATAA and ACTCGA) were significantly differently distributed in the case and control groups. Of these haplotypes, only CCACAA is associated with an increased risk of HSPN susceptibility. However, our data here indicate that four other haplotypes may protect individuals from HSPN. Additionally, the accumulation of unfavourable genotypes may increase the risk of HSPN. By comparing the HSPN and LN risk alleles, we found that A2350 is also an unfavourable genotype for LN. Finally, we found that the AG genotype (A2350G) may also increase the risk of HSPN, but may protect against LN.

\section{Acknowledgements}

This study was supported in part by the Science Foundation of Educational Commission of Heilongjiang Province (Grant No. 11551233).

\section{References}

1. Conti-Beltraminelli M, Pagani O, Ballerini G, Richetti A, Graffeo R, Ruggeri M, Forni V, Pianca S, Schonholzer C, Mainetti C, Cavalli F and Goldhirsch A: Henoch-Schönlein purpura (HSP) during treatment with anastrozole. Ann Oncol 18: 205-207, 2007.

2. Kim CJ, Woo YJ, Kook H, Choi YY, Ma JS and Hwang TJ: Henoch-Schönlein purpura nephritis associated with Epstein-Barr virus infection in twins. Pediatr Nephrol 19: 247-248, 2004.

3. Yoshioka T, Xu YX, Yoshida H, Shiraga H, Muraki T and Ito K: Deletion polymorphism of the angiotensin converting enzyme gene predicts persistent proteinuria in Henoch-Schönlein purpura nephritis. Arch Dis Child 79: 394-399, 1998.

4. Cheungpasitporn W, Jirajariyavej T, Howarth CB and Rosen RM Henoch-Schönlein purpura in an older man presenting as recta bleeding and IgA mesangioproliferative glomerulonephritis: a case report. J Med Case Rep 5: 364, 2011.
5. Rieu P and Noel LH: Henoch-Schönlein nephritis in children and adults. Morphological features and clinicopathological correlations. Ann Med Interne (Paris) 150: 151-159, 1999.

6. Ozkaya O, Soylemezoglu O, Gonen S, Misirlioglu M, Tuncer S, Kalman S, Buyan N and Hasanoglu E: Renin-angiotensin system gene polymorphisms: association with susceptibility to HenochSchönlein purpura and renal involvement. Clin Rheumatol 25: 861-865, 2006.

7. Zhou J, Tian X and Xu Q: Angiotensin-converting enzyme gene insertion/deletion polymorphism in children with HenochSchönlein purpua nephritis. J Huazhong Univ Sci Technolog Med Sci 24: 158-161, 2004.

8. Amoroso A, Danek G, Vatta S, Crovella S, Berrino M, Guarrera S, Fasano ME, Mazzola G, Amore A, Gianoglio B, Peruzzi L and Coppo R: Polymorphisms in angiotensin-converting enzyme gene and severity of renal disease in Henoch-Schöenlein patients. Italian Group of Renal Immunopathology. Nephrol Dial Transplant 13: 3184-3188, 1998.

9. Kawasaki Y: The pathogenesis and treatment of pediatric Henoch-Schönlein purpura nephritis. Clin Exp Nephrol 15: 648-657, 2011.

10. Lau KK, Suzuki H, Novak J and Wyatt RJ: Pathogenesis of Henoch-Schönlein purpura nephritis. Pediatr Nephrol 25: 19-26, 2010.

11. Sprovieri SR and Sens YA: Polymorphisms of the renin-angiotensin system genes in Brazilian patients with lupus nephropathy. Lupus 14: 356-362, 2005.

12. Tanaka R, Iijima K, Murakami R, Koide M, Nakamura $H$ and Yoshikawa N: ACE gene polymorphism in childhood IgA nephropathy: association with clinicopathologic findings. Am J Kidney Dis 31: 774-779, 1998.

13. Rodriguez-Perez JC, Macias-Reyes A, Jimenez-Sosa A, Companioni O, Rodriguez-Esparragon FJ, Cobo MA, Checa-Andres MD, Palop-Cubillo L, Alonso A and Torres A: A synergistic association of ACE I/D and eNOS G894T gene variants with the progression of immunoglobulin A nephropathy - a pilot study. Am J Nephrol 30: 303-309, 2009.

14. Qin YH, Zhou TB, Su LN, Lei FY, Huang WF and Zhao YJ: Association between ACE polymorphism and risk of $\operatorname{Ig} \mathrm{A}$ nephropathy: A meta-analysis. J Renin Angiotensin Aldosterone Syst 12: 215-223, 2011.

15. Pereira AC, Paulo M, Araujo AV, Rodrigues GJ and Bendhack LM: Nitric oxide synthesis and biological functions of nitric oxide released from ruthenium compounds. Braz J Med Biol Res 44: 947-957, 2011.

16. Wu M and Tsirka SE: Endothelial NOS-deficient mice reveal dual roles for nitric oxide during experimental autoimmune encephalomyelitis. Glia 57: 1204-1215, 2009.

17. Wanchu A, Khullar M, Deodhar SD, Bambery P and Sud A: Nitric oxide synthesis is increased in patients with systemic lupus erythematosus. Rheumatol Int 18: 41-43, 1998.

18. Crispin JC, Liossis SN, Kis-Toth K, Lieberman LA, Kyttaris VC, Juang YT and Tsokos GC: Pathogenesis of human systemic lupus erythematosus: recent advances. Trends Mol Med 16: 47-57, 2010.

19. Mok CC and Lau CS: Pathogenesis of systemic lupus erythematosus. J Clin Pathol 56: 481-490, 2003.

20. Li X, An J, Guo R, Jin Z, Li Y, Zhao Y, Lu F, Lian H, Liu P and Jin X: Association of the genetic polymorphisms of the ACE gene and the eNOS gene with lupus nephropathy in northern Chinese population. BMC Med Genet 11: 94, 2010.

21. Barreras A and Gurk-Turner C: Angiotensin II receptor blockers. Proc (Bayl Univ Med Cent) 16: 123-126, 2003.

22. Wang XL and Wang J: Endothelial nitric oxide synthase gene sequence variations and vascular disease. Mol Genet Metab 70: 241-251, 2000

23. Brenol CV, Chies JA, Brenol JC, Monticielo OA, Franciscatto P, Birriel F, Neves AG and Xavier RM: Endothelial nitric oxide synthase T-786C polymorphism in rheumatoid arthritis: association with extraarticular manifestations. Clin Rheumatol 28: 201-205, 2009. 